\title{
Hernia repair: the search for ideal meshes
}

\author{
S. Bringman $\cdot$ J. Conze $\cdot$ D. Cuccurullo $\cdot$ J. Deprest $\cdot$ \\ K. Junge $\cdot$ B. Klosterhalfen · E. Parra-Davila • \\ B. Ramshaw $\cdot$ V. Schumpelick
}

Received: 1 May 2009/Accepted: 6 November 2009/Published online: 11 December 2009

(C) The Author(s) 2009. This article is published with open access at Springerlink.com

\begin{abstract}
Background Effective repair of hernia is a difficult task. There have been many advances in hernia repair techniques over the past 50 years, but new strategies must be considered to enhance the success of herniorrhaphy.

Discussion At the 30th International Congress of the European Hernia Society, nine experts in hernia repair and experimental mesh evaluation participated in a roundtable discussion about today's unmet needs in hernia repair, including what constitutes an "ideal" hernia repair and the portfolio of "ideal" mesh prostheses. Defining characteristics of lightweight mesh, mesh alternatives, the surgeon's
\end{abstract}

S. Bringman $(\bowtie)$

Department of Surgery, Clintec, Karolinska Institutet,

Södertälje Hospital, 15286 Södertälje, Sweden

e-mail: Sven.Bringman@ki.se

J. Conze

Chirurgische Universitätsklinik u. Poliklinik der RWTH Aachen,

Pauwelsstrasse 30, 52074 Aachen, Germany

e-mail: jconze@ukaachen.de

D. Cuccurullo

Department of General and Laparoscopic Surgery,

Monaldi Hospital, Via Cilea 136, 80127 Naples, Italy

e-mail: Diego.Cuccurullo@ospedalemonaldi.it

\section{J. Deprest}

Pelvic Floor Unit, University Hospital Gasthuisberg,

Katholieke Universiteit Leuven, 3000 Leuven, Belgium

e-mail: Jan.Deprest@uzleuven.be

\section{K. Junge}

Department of Surgery, RWTH Aachen,

University Hospital Aachen, Pauwelsstraße 30,

52074 Aachen, Germany

e-mail: kjunge@ukaachen.de

role in hernia repair, adverse events, the unmet requirements for today's hernia repair, and optimized animal models were among the topics discussed.

Conclusion The ideal mesh's construction is still in progress, but greater understanding of its critical characteristics was explored. It is hoped that these suggestions will lead to the development of improved hernia treatments and a maximally effective portfolio of hernia mesh prostheses.

Keywords Surgical mesh · Prosthesis design · Complications - Operative surgical procedure . Animal models

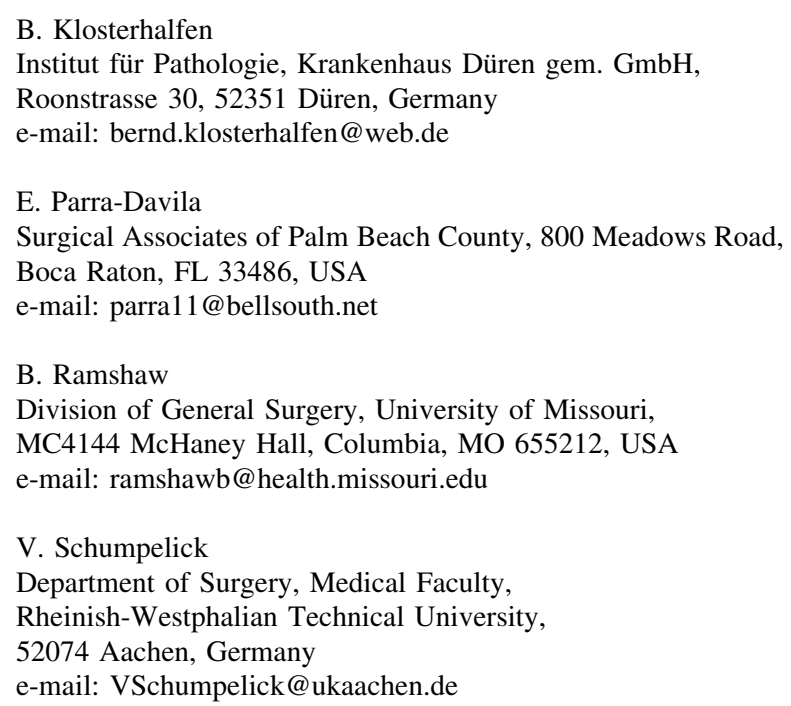




\section{Introduction}

As surgeons gain experience caring for hernia repair patients, techniques continue to evolve and clinical outcomes continue to improve. Surgical repair of hernias is one of the most common operative procedures performed, and there is no single gold-standard operative technique in hernia repair [1]. The decisions about which techniques to use are not well defined, and instead the choice depends on tradition, context, and familiarity with the type of hernia.

The debut of the first mesh indicated for hernia repair was in 1958 with the introduction of polyethylene mesh by Usher et al. [2]. The use of synthetic meshes was a milestone in hernia repair and led to the development of many other mesh products of various polymer types, densities, and elasticity. Since the introduction of polyethylene mesh, there has been an increased reliance on the use of mesh in hernia repair, either for bridging the defect or for reinforcing the abdominal wall. Meanwhile, identifying the right mesh for the right patient can be a difficult, if not impossible, task. At the 30th International Congress of the European Hernia Society, nine international experts in hernia repair and experimental mesh evaluation participated in a roundtable discussion about today's unmet needs for hernia repair, including defining optimal hernia repair outcomes and the portfolio of "ideal" mesh prostheses.

\section{Discussion}

Redefining the term "lightweight" mesh

Understanding the technical and general definition of "lightweight" and "heavyweight" mesh can be difficult as there are no official definitions or consensus on classifications. The panel evaluated connotations associated with existing lightweight hernia mesh nomenclature and discussed the technical aspects to approach a working definition.

Most surgeons in the United States have had few problems with heavyweight mesh, reporting few recurrences and complications. Interestingly, there is a mismatch between this clinical experience and published clinical data. Heavyweight meshes have a higher tensile strength and are stronger than lightweight meshes [3]. The use of heavyweight meshes is associated with increased complications and adverse events, such as fistula and adhesion formation and pain [4, 5]. Although these complications are mainly observed during intraperitoneal application, they have also been observed as a result of extraperitoneal placement [6]. Heavyweight meshes have an increased surface area and, therefore, produce a more intense foreign body reaction. They also tend to shrink more than lightweight meshes and are stiffer, which can make normal abdominal movements difficult or unnatural [7].

The panel agreed that today the term "lightweight" is no more than a name alluding to the recent history of marketed meshes. The term "lightweight" is not always simply descriptive of the product being low in weight, nor can it be simply defined by a cutoff value of weight per square meter, filament, or specific pore size. Lightweight typically refers more to meshes with a larger pore size, resulting in a smaller surface area. The lower amount of material present in lightweight meshes should lead to decreased foreign body reaction and fibrosis [8]. Conceptually, lightweight meshes are more flexible, both before and after tissue integration, which should improve physical properties, allowing a better activity profile post-surgery [3]. This is most likely not at the expense of the strength of the repair, as the type of nonabsorbable synthetic mesh used for hernia repair is not related to recurrence rates [4]. Conversely, implant rigidity of heavyweight mesh has led to greater complications and adverse events, such as pain and foreign body reactions.

Although the panelists agreed that current lightweight meshes have a larger pore size with a smaller surface area and are more flexible than heavyweight mesh, they could not agree on the specifications for the characteristics. Furthermore, the term "lightweight" is used extensively in the literature with no official definition. Recently, Hollinsky et al. [3] performed biomechanical studies on lightweight versus heavyweight mesh. Thickness and weight were used to distinguish lightweight $(<0.5 \mathrm{~mm}$ thick, $<1 \mathrm{~g})$ from heavyweight $(>0.5 \mathrm{~mm}$ thick, $>1 \mathrm{~g})$ for mesh measuring $15 \times 10 \mathrm{~cm}$. However, the pore size overlapped between groups (1.24-3.0 mm for lightweight, $<1-2.0 \mathrm{~mm}$ for heavyweight). While the Holinsky article presents a good basis for characterizing lightweight and heavyweight meshes, the panelists believed that pore size is the most important determinant. Throughout this article, the panelists use the term "lightweight" based on their own opinions. It is recommended that the appropriate organizations determine the characteristics for each mesh. In other words, there is an urgent need for further standardization of mesh classification beyond that already available [9].

Identifying needs in today's hernia repair

Looking beyond the features and benefits of meshes to discuss the key needs and expected results of hernia repair, it is necessary to understand the pathology of hernia. Quality of collagen, for example, is important in the pathophysiology of hernias and plays an important role in repair [10]. The purpose of surgical meshes in hernia repair is to reinforce and replace tissue for long-term 
stabilization of the abdominal wall. There are different requirements for inguinal repairs versus ventral repairs, small hernias compared with large hernias, and open approaches versus laparoscopic techniques. Small hernias can be repaired with most mesh types, whereas it is much more difficult to find an effective mesh for larger hernias. For pelvic floor hernias, one panellist recommended a mesh that is more elastic and less prone to erosion. Meshes that produce strong foreign body reactions can result in a large number of complications, including fibrosis and pain [4]. Further, there tends to be an increased prevalence of nerve entrapment with the use of heavyweight mesh in inguinal repairs [4].

An ideal portfolio of meshes would have the benefits of both heavyweight and lightweight meshes, such as the strength of a heavyweight mesh and the flexibility of a lightweight mesh with none of the adverse events.

One aspect of all mesh repairs that needs improvement is attachment to the abdominal wall. Current meshes on the market could be faster and easier to implant, whether deployed laparoscopically or through an open incision. This expediency would lead to shorter operating times, appealing to both the patient and the surgeon. The panelists agreed that more than one mesh type is required to accommodate the many possible patient/situation needs. Mesh requirements depend on technique and mesh position with respect to the abdominal wall (extraperitoneal versus intraperitoneal). The surgeon should have the ability to choose a mesh device suited to each patient's particular hernia condition.

The "ideal" mesh for hernia repair: defining attributes and characteristics

Mesh development is an ongoing process, reflecting changes in polymer structure, biocompatibility, operative handling, and cost. So what does the "ideal" portfolio of meshes resemble? The panel's conversation began to distinguish the mesh characteristics according to different surgical needs, but generally the mesh features desired in four common surgical procedures (open ventral, open inguinal, laparoscopic ventral, and laparoscopic inguinal hernia repairs) were very similar. The topics discussed were pore size, geometry, active surface area, mesh memory, affinity for water, elasticity, and polymer type. According to the panel, a good mesh will have negligible foreign body reaction with no pathologic fibrosis.

The panel pointed out that pore size can affect adhesions following intraabdominal placement, tissue integration, active surface area, elasticity, and memory. For open ventral hernia repair, pore size was discussed extensively. The heavyweight microporous meshes have a lower risk of tissue-to-mesh adhesion but carry a risk of encapsulation and foreign body reaction, resulting in decreased integration. Lightweight macroporous mesh results in better tissue in-growth and lower foreign body reaction but may lead to a higher risk of adhesions [11]. A larger pore size also provides optimal flexibility for improved physical properties, allowing a better activity profile post-surgery, but relinquishes memory, which is important for handling during the procedure. Despite the absence of a formal approval on package inserts for many synthetic mesh products, the panel agreed that larger pore meshes seem to be safe to use in contaminated environments. The panel also agreed that a monofilament mesh with a pore size of $>2.5 \mathrm{~mm}$ seems ideal, although some authors recommended pore sizes as large as 3 and $6 \mathrm{~mm}$.

In all hernia repair techniques, a strong mesh is important for augmentation of the abdominal wall and to prevent recurrences. The panel agreed that a tensile strength of $16 \mathrm{~N}$ was probably more than sufficient to augment the abdominal wall; for bridging of large defects, an increased tensile strength of $32 \mathrm{~N}$ may be necessary. The panel concluded that mesh should be flexible but also have a good memory, and it should have elasticity in more than one dimension, allowing it to stretch in more than one direction and then return to its original shape. In this way, the mesh should match the abdominal wall dynamics as closely as possible. Flexibility and memory, which make a mesh more adaptable, are also important to optimize the surgical handling of the mesh. The mesh should have an adequate adhesive quality that requires minimal or no additional fixation, even for large defects.

In both laparoscopic inguinal and ventral procedures, the need for a mesh that is flexible with good memory was emphasized. In open inguinal Lichtenstein repairs, a mesh with improved adhesive properties, especially at the ends, is seen as optimal. For laparoscopic ventral hernia repairs, the mesh composition needs to form an adequate barrier between the viscera and the mesh. Although tissue separation properties are not required in other hernia repairs, the panel agreed that mesh used in intraabdominal mesh repair should have a solid absorbable barrier that prevents adhesion to the intestines. As adhesions begin to form immediately, the barrier should absorb slowly enough to prevent formation of adhesions. This is complicated by the fact that adhesions have no definite time dynamic [12]. The panel also concluded that the parietal fraction of the mesh should have a good capacity for in-growth, and the construction should be strong enough for adequate bridging and augmentation.

Microporous materials with a strong affinity for water have been developed to reduce the risk of adhesion formation, but these materials lack adequate tissue integration and are more likely to cause recurrence [13]. As a result, composite meshes were developed with a smooth 
microporous surface on one side for contact with the viscera, with the other side designed for appropriate tissue integration [13]. According to the panel, an ideal mesh would be a monofilament mesh that would prevent adhesions yet still enable growth of the adjacent tissue for optimal augmentation.

The desire for a transparent mesh for use in all hernia repair techniques was also discussed. Transparency would assist proper placement of the mesh and possibly lead to a decrease in recurrence rates, pain, and other possible adverse effects, potentially leading to improved quality of life. In some institutions, surgeons seldom have their choice of mesh products available for use in hernia repairs. In these cases, the meshes stocked in the operating room reflect the least expensive options on the market. So, in order to complete the theoretical construction of the "ideal" mesh, prices should be reasonable.

\section{Alternatives to mesh}

While mesh was the primary discussion topic, the panel addressed alternatives as well. Myofascial transposition, in which lateral components are separated, is a technique popularized over the past 10-15 years [14]. This more functional repair tends to have high recurrence rates and is, therefore, primarily reserved for massive midline hernias in which currently available meshes are inadequate. Absorbable biological and biosynthetic materials are not often used and have not lived up to initial expectations. These materials are also associated with high failure rates, not only in abdominal and inguinal hernia repair, but also in pelvic floor hernia repairs $[15,16]$. Long-term outcomes are unknown. Therefore, these materials are reserved for situations in which mesh is not appropriate, such as in septic conditions, and even then they might be prone to recurrence and graft-related complications. While the future of these biological materials looks hopeful, the currently available products are not yet adequate for successful hernia repair.

Establishing animal models for the development of the "ideal" mesh

The panel concluded that animal models resembling the human hernia are a useful tool for researchers to investigate hernia treatment options. The current animal models used to study hernia repair are not perfect, and panel members have had hands-on experience with a variety of these. From their experiences, the panelists agreed that artificially created hernias in animals are poor hernia models as they do not truly recreate the biological defects that cause hernias, such as collagen defects. Furthermore, the defects that are created to test mesh products are not real-world defects that surgeons would encounter. In order to serve as a useful model, the pathology in the animal must be similar to the human hernia equivalent. One factor when considering an animal model is similarities in the elasticity of the abdominal wall. Although the panel did not reach a consensus for the most appropriate test or animal model, they agreed that animal models are useful when comparing different meshes in the same species either in vivo or ex vivo. Studies in humans and large animals are the only way that most issues, such as elasticity, chronic pain, foreign body reaction, and adhesion, will be observed.

What problems animal models can solve and which animals are the most appropriate for use will differ depending on the purpose of the study. Animal models currently used include rodents, rabbits, pigs, and primates. Small animals or even cell cultures are instructive for studying the inflammatory reaction and biocompatibility, but for abdominal wall function and elasticity, larger animals are more suitable [17]. The panel agreed that although ineffective for other comparisons, pigs are useful to simulate mesh implantation within the human body as pigs have a similar body size to humans, and further work is being conducted to recreate human collagen problems in swine. Sheep and rabbits are reasonable models to mimic vaginal operating conditions; potentially, they are also useful models for pelvic floor damage due to pregnancy and birth, although the latter has not been formally studied yet.

Defining optimal clinical outcomes and limiting adverse events

The panel agreed that the two most important outcome measures are recurrence and pain. Chronic pain is an issue that primarily affects patient quality of life. For these patients, it is difficult to imagine a more imperative issue. According to some studies, chronic pain can occur in up to $40 \%$ of hernia surgeries [18]. The panel agreed that chronic pain, stiff abdomen, and foreign body sensation are least often observed with the use of a lightweight mesh and a laparoscopic approach $[4,8,19]$.

A truly successful hernia repair requires effective bridging or augmentation that will prevent recurrence, but the panel agreed that complete avoidance of recurrence is unlikely. Recurrence rates tend to be higher in patients who are obese, smoke, are older, have anemia, or are male [20, 21]. If reoperation is required in the event of a recurrence, the incidence of chronic pain increases. There seem to be inconsistencies in the way recurrences are reported, especially in clinical trials, which affects the available clinical data. A recurrence may be anatomical but not functional, or it may be both. It is possible to have an asymptomatic or not visible recurrence, which may only be seen during 
ultrasound examinations (subclinical). Clinical trials that do not use ultrasound potentially miss some recurrences that are not clinically obvious.

Secondary to chronic pain and recurrence are foreign body reactions, seroma formation, quality of life with a return to normal activity, infection, comfort, and cosmesis with minimal scarring. A return to normal activity levels is an important clinical outcome measurement. A successful procedure is one in which a patient has no sensation of the foreign body, no stiffness, and can return quickly to normal activity.

According to the panel, quality of life and comfort are influenced by many factors and should be measured before and after surgery. Several questionnaires are available to help determine quality of life. The Carolina Comfort Scale and the Swedish Pain Questionnaire are specifically used for patients with hernias, and the 36-Item Short Form (SF36 ) is used as a measure of general well-being [22, 23]. Although these tests are useful, the panel agreed that a better questionnaire is needed.

Both superficial wound infections and mesh infections should be distinguished and avoided [24]. Foreign body reactions, such as seromas, can present more than 1 year after surgery. Tissue mobilization is important to prevent seroma formation, and binders may help reduce fluid formation. Seroma formations are common in laparoscopic procedures, and the panelists agreed, based on their own experiences (and from published data), that lightweight meshes result in fewer seromas [25, 26].

The hernia repair process should begin with informing the patient about the procedure, signs and symptoms of infection, and recurrence, and how to distinguish between normal or abnormal pain and discomfort. Following patient education, technique should be considered, which is the most important factor of success. Most surgeons are hesitant to modify their surgical methodology but understand that technical advances, such as mesh repair, are important to include into practice. The panelists agreed that in the groin, the right technique can minimize or completely prevent pain. The ideal technique includes a high-quality dissection, maximum visualization, maximum coverage of the anatomical defect using wide overlap, and minimum fixation. The procedure should consider both the physiology of the hernia region and the integrity of the spermatic cord. In order to eliminate chronic pain and stiffness, permanent fixation should ideally be avoided or at least be as minimal as possible [4].

A return to normal physiology and maintenance of the repair is the postoperative goal. Optimal mesh flexibility and elasticity ensure a quick return to function, and heavyweight mesh should be avoided as it can cause chronic pain due to retraction and tends to reduce the ability of the abdominal wall to move in a normal fashion
[27]. Good cosmesis with no seroma formation and minimal scarring are ideal for both the patient and the surgeon. The optimal hernia repair will result in minimal adverse events, such as pain, foreign body reaction, and seroma formations. The panel concluded that an optimal clinical outcome is a repair that is free from recurrence, pain, and infection, with minimal scarring and with improvement in patient's quality of life.

The surgeon's role and responsibilities in the complete management of hernia repair

The role and responsibilities of the surgeon with regard to training, experience, and interaction with the patient are important to understand if outcomes are to be optimized. Surgical residency training with mentors who are experienced in hernia repair or in hospitals that perform large numbers of herniorrhaphies is necessary to ensure a comprehensive understanding of the anatomy and proper technique. Even with experience and good operative technique, there will still be issues that the surgeon cannot control. Residency training today is insufficient, and residents should be exposed to more cases to enhance their education. Fellowships for minimally invasive surgery can improve the skills of commonly performed surgeries, including hernia repair, and are a good option for surgeons who are leaving residency programs. The prevalence of pelvic floor prolapse problems is increasing, and more gynecologic surgeons are needed with the skills to perform these repairs. Due to the increasing number of pelvic floor hernias being performed, the procedure is becoming more standardized. Most hernia repairs are performed by general surgeons and have good results, but there are still improvements that can be made to address the unmet educational needs of surgeons. While the number of hernia repair centers is increasing in the United States, the constructs of socialized medicine often preclude other countries from creating such arrangements.

Deciding whether or not to use mesh for hernia repair can be a difficult task. With the advent of laparoscopy, the use of mesh has become commonplace for hernia repair and is increasingly popular for use even in young patients. The use of a laparoscopic technique inherently means a mesh will be employed. Furthermore, in open repair of incisional hernias, the use of mesh is recommended. Even using surgical mesh with a Lichtenstein repair is a technique gaining in popularity in less developed countries. According to the Swedish Hernia Registry, high recurrence rates have been reported in hernia operations performed without mesh, suggesting that mesh should always be used. Patient involvement is encouraged if the patient is properly educated and can help inform the decision of using mesh. This involvement should be documented in the event of 
complications. Ultimately, the choice of whether or not to use a surgical mesh is the surgeon's prerogative. Age, smoking status, pre-existing medical conditions, and patient activity level are all factors that need to be considered when making this determination.

\section{Conclusions}

The development of mesh prosthetics for successful hernia repair has proven to be an intricate and difficult task. Many important lessons have been learned from both successful and unsuccessful herniorrhaphies. To move forward into a new era of hernia mesh prosthetics, the panel agreed that new technologies and novel approaches must be investigated and designed. The portfolio of meshes should be cost effective; have no adhesion potential, excellent tissue integration, minimal shrinkage, good memory; and be easy to use. Ideal meshes should not promote infection, fistula, or seroma formation and should not limit or negatively affect a patient's normal activity.

The panel concluded that a new quality-of-life survey should be developed. Additionally, present-day teaching and awareness programs should be further expanded, including enhanced professional education for surgical residents as well as education of patients. The use of animal testing in hernia repair clinical trials is presently inadequate, and better animal models should be developed to advance today's methodologies. Certain topics for future roundtable discussions were identified, such as mesh properties and fixation, disseminating information to hernia patients, training in hernia surgery, and quality-of-life scales for evaluating hernia surgery. Roundtable meetings on these topics are important, and outcomes of such meetings would be of interest to many hernia surgeons.

These proceedings provided insight into the features and benefits of innovation for the portfolio of ideal hernia repair prostheses and into how such attributes translate into better clinical patient outcomes. There is optimism that continued discussion can lead to the development of new and "ideal" hernia mesh prostheses, which would improve the outcomes of hernia repair procedures.

Acknowledgments The authors take full responsibility for the content of the paper but thank Caudex Medical (supported by Ethicon, Inc.) for editorial assistance.

Disclosures S.B. has participated in courses, workshops, and advisory boards organized by Ethicon, Inc. J.C. has participated at either courses, panel meetings, workshops and/or advisory boards organized by Ethicon, Inc., Lifecell, BBraun, and Covedien. D.C. has nothing to disclose. J.D. has received unrestricted grants for experimental studies from Bard, American Medical Systems, and Ethicon, Inc., and a grant for a clinical study from American Medical Systems. K.J. has participated at courses and workshops regarding hernia repair organized by Ethicon, Inc. B.K. has nothing to disclose. E.P.D. has participated in courses and workshops regarding hernia repair organized by Ethicon, Inc. B.R. has received grants, honoraria or fellowship support from WL Gore, and Covidien, Ethicon Endo-surgery, Inc., Ethicon, Inc., MTF, Atrium, Lifecell. V.S. has nothing to disclose.

Open Access This article is distributed under the terms of the Creative Commons Attribution Noncommercial License which permits any noncommercial use, distribution, and reproduction in any medium, provided the original author(s) and source are credited.

\section{References}

1. Rutkow IM (2003) Demographic and socioeconomic aspects of hernia repair in the United States in 2003. Surg Clin North Am 83:1045-1051, v-vi

2. Usher FC, Ochsner J, Tuttle LL Jr (1958) Use of Marlex mesh in the repair of incisional hernias. Am Surg 24:969-974

3. Hollinsky C, Sandberg S, Koch T, Seidler S (2008) Biomechanical properties of lightweight versus heavyweight meshes for laparoscopic inguinal hernia repair and their impact on recurrence rates. Surg Endosc 22:2679-2685

4. Klosterhalfen B, Junge K, Klinge U (2005) The lightweight and large porous mesh concept for hernia repair. Expert Rev Med Devices 2:103-117

5. Miller K, Junger W (1997) Ileocutaneous fistula formation following laparoscopic polypropylene mesh hernia repair. Surg Endosc 11:772-773

6. Farmer L, Ayoub M, Warejcka D et al (1998) Adhesion formation after intraperitoneal and extraperitoneal implantation of polypropylene mesh. Am Surg 64:144-146

7. Amid PK (2004) Shrinkage: fake or fact? In: Schumpelick V, Nyhus LM (eds) Meshes: benefits and risks. Springer, Berlin

8. Klosterhalfen B, Klinge U, Schumpelick V (1998) Functional and morphological evaluation of different polypropylene-mesh modifications for abdominal wall repair. Biomaterials 19:2235-2246

9. Amid PK (1997) Classification of biomaterials and their related complications in abdominal wall hernia surgery. Hernia 1:15-21

10. Junge K, Klinge U, Rosch R et al (2004) Decreased collagen type I/III ratio in patients with recurring hernia after implantation of alloplastic prostheses. Langenbecks Arch Surg 389:17-22

11. Eriksen JR, Gogenur I, Rosenberg J (2007) Choice of mesh for laparoscopic ventral hernia repair. Hernia 11:481-492

12. Conze J, Schumpelick V (2004) Prevention of adhesion-just an illusion? In: Schumpelick V, Nyhus LM (eds) Meshes: benefits and risks. Springer, Berlin

13. Arnaud JP, Hennekinne-Mucci S, Pessaux P et al (2003) Ultrasound detection of visceral adhesion after intraperitoneal ventral hernia treatment: a comparative study of protected versus unprotected meshes. Hernia 7:85-88

14. Espinosa-de-Los-Monteros A, de la Torre JI, Ahumada LA et al (2006) Reconstruction of the abdominal wall for incisional hernia repair. Am J Surg 191:173-177

15. Blatnik J, Jin J, Rosen M (2008) Abdominal hernia repair with bridging acellular dermal matrix — an expensive hernia sac. Am J Surg 196:47-50

16. Deprest J, De Ridder D, Roovers JP et al (2009) Medium term outcome of laparoscopic sacrocolpopexy with xenografts compared to synthetic grafts. J Urol 182:2362-2368

17. Penttinen R, Gronroos JM (2008) Mesh repair of common abdominal hernias: a review on experimental and clinical studies. Hernia 12:337-344 
18. Koninger J, Redecke J, Butters M (2004) Chronic pain after hernia repair: a randomized trial comparing Shouldice, Lichtenstein and TAPP. Langenbecks Arch Surg 389:361-365

19. Klinge U, Müller M, Brücker C, Schumpelick V (1998) Application of three dimensional stereography to assess abdominal wall mobility. Hernia 2:11-14

20. Junge K, Rosch R, Klinge U (2006) Risk factors related to recurrence in inguinal hernia repair: a retrospective analysis. Hernia 10:309-315

21. Jonsson K, Jensen JA, Goodson WH 3rd et al (1991) Tissue oxygenation, anemia, and perfusion in relation to wound healing in surgical patients. Ann Surg 214:605-613

22. Heniford BT, Walters AL, Lincourt AE et al (2008) Comparison of generic versus specific quality-of-life scales for mesh hernia repairs. J Am Coll Surg 206:638-644

23. Haapaniemi S, Nilsson E (2002) Recurrence and pain three years after groin hernia repair. Validation of postal questionnaire and selective physical examination as a method of follow-up. Eur $\mathrm{J}$ Surg 168:22-28

24. Jezupovs A, Mihelsons M (2006) The analysis of infection after polypropylene mesh repair of abdominal wall hernia. World $\mathrm{J}$ Surg 30:2270-2278 (discussion 2279-2280)

25. Lim C, Basson H, Jayasundera M (2008) A comparison of laparoscopic and open inguinal hernia repair. ANZ J Surg 78:A48A60

26. Horstmann R, Hellwig M, Classen C et al (2006) Impact of polypropylene amount on functional outcome and quality of life after inguinal hernia repair by the TAPP procedure using pure, mixed, and titanium-coated meshes. World J Surg 30:17421749

27. Cobb WS, Kercher KW, Heniford BT (2005) The argument for lightweight polypropylene mesh in hernia repair. Surg Innov 12:63-69 